\title{
Desenvolvimento de uma solução de comunicação alternativa para dispositivos móveis para crianças com distúrbios de comunicação baseada em normas de acessibilidade
}

\author{
Mauro José Conte ${ }^{1}$, Fabiana de Melo G. Garcez Garcez ${ }^{2}$, Alejandro R. Garcia \\ Ramirez ${ }^{1}$ \\ ${ }^{1}$ Departamento de Computação Aplicada - Universidade do Vale de Itajaí (SC) \\ Rua Uruguai, 458, Itajaí - SC - Brazil \\ ${ }^{2}$ APAE - Florianópolis - (SC). Itacorubi, Florianópolis. SC. Brazil. \\ \{maurojconte, garcia.ramirez\}@gmail.com, fabi.garcez@hotmail.com
}

\begin{abstract}
Resumo. Os comitês do Consórcio World Wide Web-W3C, através das Melhores Práticas para Web Móvel - MWBP, geram continuamente recomendações de acessibilidade que visam melhorar a experiência do usuário na hora de acessar conteúdos em dispositivos móveis. No caso particular das pessoas com deficiência, essas recomendações visam também reduzir o impacto ocasionado pela deficiência. No entanto, constata-se que uma boa parte das soluções existentes não levam em conta esses princípios no desenvolvimento de produtos para esse público. Este trabalho apresenta o desenvolvimento de um aplicativo para tablet, baseado nos princípios da Comunicação Aumentativa e Alternativa (CAA), levando em conta as recomendações de acessibilidade preconizadas pela $M W B P$, com o objetivo maior de atuar na Educação Especial, contribuindo no processo de avaliação da deficiência intelectual e também no processo de comunicação de crianças que apresentam distúrbios da fala. Cabe destacar que a metodologia de desenvolvimento seguiu o princípio de Projeto Centrado no Usuário, motivo pelo qual o desenvolvimento e avaliação do aplicativo foram realizados em conjunto com profissionais da APAE de Florianópolis.
\end{abstract}

Abstract.The World Wide Web - W3C Consortium committee, through the Mobile Web Best Practices - MWBP, continuously generate accessibility guidelines aimed at improving the user experience when accessing content on mobile devices. Concerning to disabled people, these recommendations also aim to reduce the impact caused by the deficiency. However, it appears that much of the existing solutions do not take into account these principles when developing solutions for this audience. This paper presents the development of a tablet solution based on the principles of the Augmentative and Alternative Communication (AAC) and takes into account the accessibility guidelines recommended by MWBP. The app is intended for Special Education, contributing to evaluate the intellectual impairment of children who have speech disorders. The methodology followed the principle of the User Centered Design, so the development, application and evaluation were carried out in conjunction with professionals of the APAE Florianópolis. 


\section{CBIE-LACLO 2015}

Anais dos Workshops do IV Congresso Brasileiro de Informática na Educação (CBIE 2015)

\section{Cenário de Uso / Informações Gerais}

Este trabalho aborda o desenvolvimento de uma solução de CAA focada na elaboração de conteúdos para dispositivos móveis (tablet), apresentados através de uma interface de homem-máquina que adota padrões de interação e acessibilidade para usuários que apresentam deficiência intelectual e distúrbios de comunicação. Envolve a apresentação e organização dos conteúdos do aplicativo, assim como o uso de recursos de forma otimizada, de modo a atender às necessidades dos usuários no ambiente móvel.

O aplicativo possui, como fim maior, contribuir na avaliação do tipo de deficiência intelectual das crianças atendidas nas APAEs, podendo também ser utilizado em atividades de comunicação alternativa para tratar também a deficiência na fala. Cabe destacar que este trabalho é realizado em parceria com uma equipe multidisciplinar da APAE - Florianópolis, onde participam pedagogos, fonoaudiólogos, psicólogos e terapeutas ocupacionais.

A interface do aplicativo foi baseada no conhecimento sobre o público-alvo e também nos fundamentos de acessibilidade para dispositivos móveis, realizando-se uma interação com os profissionais envolvidos durante todo o processo de desenvolvimento, de modo a verificar, na prática, se os requisitos de acessibilidade propostos possuem o impacto almejado. Cabe salientar que este trabalho visa contribuir na geração de soluções nacionais, apoiando o trabalho dos profissionais que atuam na Educação Especial.

\section{Apresentação do software}

A Comunicação Aumentativa e Alternativa - CAA é essencial para o processo educativo, propiciando a inserção social, atendendo a necessidade de fazer amigos, o desenvolvimento de habilidades acadêmicas e o engajamento nos processos comunicativos familiares e da comunidade [Cook and Polgar, 2008]. Contudo, um problema comum é o custo e disponibilidade de tais soluções [Higashi et al., 2005].

Além da barreira formada pelos custos e a disponibilidade, Doval, Carballo and Jeremías (2010) esclarecem que os usuários de sistemas de CAA possuem grande diversidade de necessidades de comunicação, de modo que uma boa solução deve àquela que resulte ser adaptável às necessidades de desenvolvimento e aprendizado do usuário, bem como às habilidades, preferências e necessidades futuras de comunicação.

Nesta linha de raciocínio, Schindler, Dygert e Nagler, (2009) afirmam que as necessidades especiais variam muito de um indivíduo para outro, dependendo de cada deficiência, e salientam a importância de que as ferramentas de CAA possam ser configuráveis. Mas, o que deve ser configurado, por quem, e como?

É nesse ponto que entram as normas de acessibilidade. Elas são diretrizes que auxiliam ao projetista de interfaces homem-computador para garantir o acesso adequado (usabilidade), aos recursos do sistema projetado, garantindo também a adaptabilidade do sistema. Salienta-se que a acessibilidade não se restringe apenas às pessoas com algum tipo de deficiência. Acessibilidade significa oportunidade de acesso e disponibilização da informação para todos os usuários, permitindo a compreensão do conteúdo, independentemente das limitações do corpo ou funcionais ou da plataforma e tecnologias utilizadas, maximizando a experiência do usuário. 


\section{CBIE-LACLO 2015}

Anais dos Workshops do IV Congresso Brasileiro de Informática na Educação (CBIE 2015)

Dessa forma, e com base na experiência adquirida em um trabalho precedente, concebido para desktop, e agora focado no desenvolvimento de soluções de CAA para dispositivos móveis, conjugado com o estudo das normas de acessibilidade do WCAG e do MWBP, foram analisadas as soluções existentes no mercado e as normas por elas atendidas (ou não atendidas), de modo a projetar uma solução que leva-se em conta recursos considerados relevantes para garantir o sucesso dessa interface computacional. Uma avaliação piloto na APAE Florianópolis permitiu verificar a validade da proposta aqui apresentada.

Esta seção apresenta os resultados do protótipo desenvolvido na linha de CAA. Em particular, são ilustrados os recursos destinados aos alunos e aos profissionais que efetuam os atendimentos na APAE, realçando suas funções.

Cabe destacar que o desenvolvimento levou em conta 15 recomendações de acessibilidade, de acordo com o Grupo de Trabalho W3C, 9 de julho de 2009, em particular as Mobile Web Best Practices [Rabin and Mccathienevile, 2008]. Junto às recomendações, consideradas relevantes, enunciam-se as características ausentes e os problemas que podem acontecer no contexto da deficiência.

\subsection{Imagens e Interface do Aplicativo}

A interface "Aluno" contém diversas telas, que guiam o trabalho do profissional. A Figura 1 apresenta a tela de um atendimento já iniciado, ilustrando as categorias disponíveis para o profissional e para o aluno.

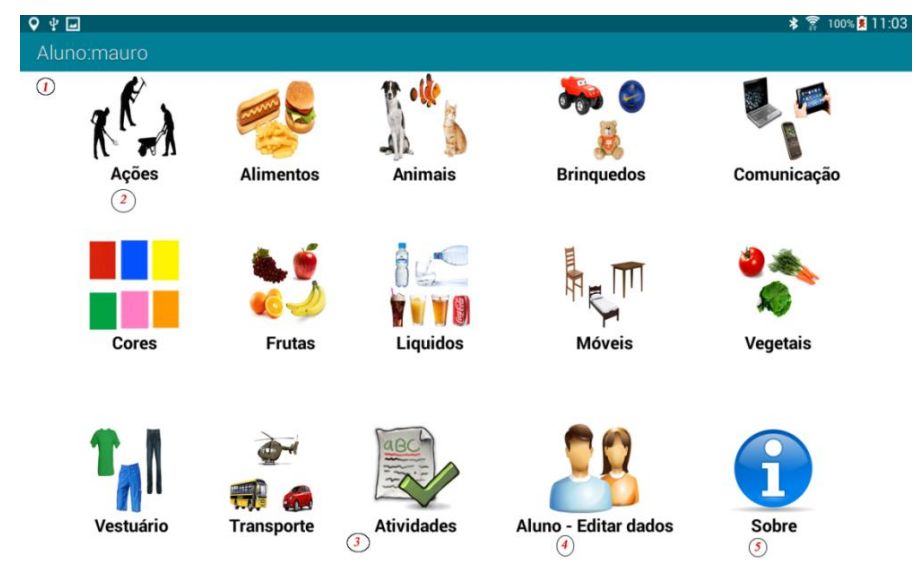

Figura 1 - Tela que ilustra os recursos do aplicativo.

Na Figura 1 foram adicionados círculos numerados para identificar melhor os recursos da interface, descritos a seguir:

1) Nesta tela, aparece o nome do aluno na barra de título. Quando o utilizador acessar essa tela e estiver habilitado o áudio, o nome do aluno será ouvido através do vocalizador.

2) São as categorias disponíveis no aplicativo, sendo, ordenadas em: Ações, Alimentos, Animais, Brinquedos, Comunicação, Cores, Frutas, Líquidos, Móveis, Vegetais, Vestuário e Transporte. Todas as imagens empregadas foram repassadas pelos profissionais da APAE - Florianópolis e convertidas empregando o tamanho e formato adequados para a aplicação. 
3) Enlace de entrada para as atividades do aplicativo. Nessa área estão presentes quatro opções de atividades, que serão descritas posteriormente.

4) Esta opção permite Editar/Consultar os dados de cada aluno cadastrado, mas não é possível incluir um novo aluno através desse enlace, apenas visualizar os dados. Acessando a interface de edição aparecerão informações do aluno (nome, tipo de diagnostico, data de nascimento, data da avaliação, observações do atendimento, foto e áudio), editados previamente pelo profissional que fez o registro do atendimento.

5) Através da última opção desta tela, intitulada 'Sobre', aparecem informações de todos os integrantes do Projeto (Aluno, Orientador, Profissionais da APAE de Florianópolis), e das instituições participantes;

A opção de "sair" para todos os formulários está disponível no próprio dispositivo.

Para cada imagem das categorias ilustradas na Figura 1 há uma interface com outras imagens dentro de cada grupo. Ver, por exemplo, a Categoria Alimentos, ilustrada na Figura 2. $\mathrm{Na}$ interface de cada grupo ou categoria o profissional pode trabalhar com a vocalização de cada imagem, gravando o áudio que for mais relevante para cada símbolo/objeto da respectiva categoria. Outra função importante dentro do formulário é a inclusão de um botão de acesso à edição dos dados do aluno para incluir informações de diagnóstico sobre o aluno (observações).

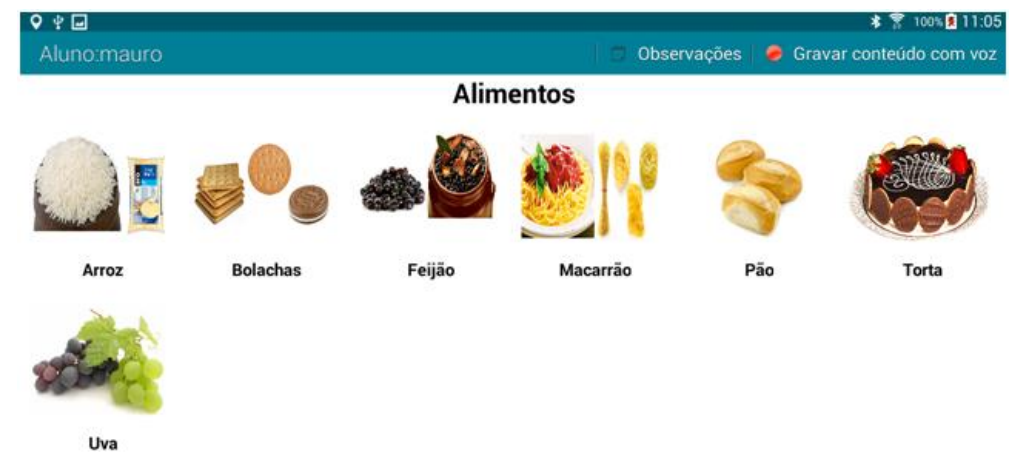

Figura 2 - Interface da categoria dos alimentos

A interface ilustrada na Figura 2 mostra os itens da categoria Alimentos, em ordem alfabética, (arroz, bolachas, feijão, macarrão, pão, torta e uva). Todas as imagens foram adaptadas levando em consideração o layout utilizado e as especificações do dispositivo móvel (tablet). A Figura 3 demonstra o que acontece quando é selecionado o item de categoria Pão, dentro da categoria dos alimentos.

Após o toque na tela da categoria de alimentos, e posterior escolha do item Pão, aparece essa interface para o aluno, reproduzindo-se o áudio previamente gravado para essa imagem. Sempre, caso necessário, o profissional pode regravar o áudio para cada imagem, embora vale realçar que, para cada imagem, apenas é associado um arquivo de áudio.

$\mathrm{Na}$ Figura 4 ilustra-se a tela inicial, que permite o acesso ao aplicativo, onde o profissional deve informar uma identificação e uma senha. 
CBIE-LACLO 2015

Anais dos Workshops do IV Congresso Brasileiro de Informática na Educação (CBIE 2015)

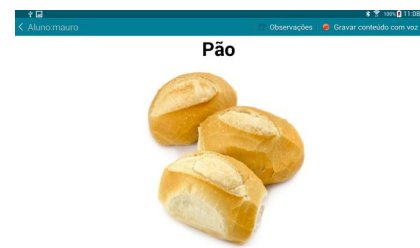

Figura 3- Interface da categoria dos alimentos - Pão.

Após o primeiro acesso ele pode optar por deixar armazenada a senha no dispositivo, bastando marcar a opção Manter Conectado e, sempre que for executado novamente o aplicativo, não será mais necessário informar novamente a identificação.

Cabe destacar que no banco de dados do dispositivo são armazenadas as informações dos alunos, envolvendo os dados pessoais, que devem ser tratados com toda a integridade e segurança necessária.

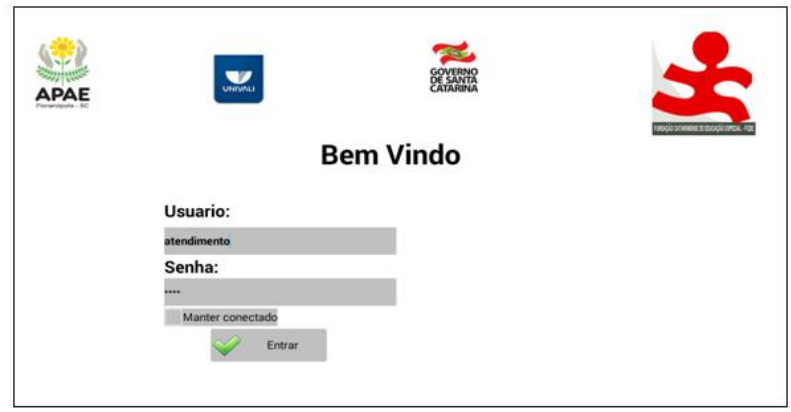

Figura 4-Interface para o controle de acesso ao aplicativo.

A Figura 5 ilustra a interface onde aparecerão os alunos cadastrados no aplicativo. Cabe salientar que todas as interfaces do aplicativo possuem conteúdo textual e não textual (imagens, áudio). Caso o aluno não tenha sido cadastrado previamente, deve selecionar-se a opção Aluno - Editar Dados (ver Figura 1). Salientase que o cadastro é necessário para poder usar os recursos do aplicativo.

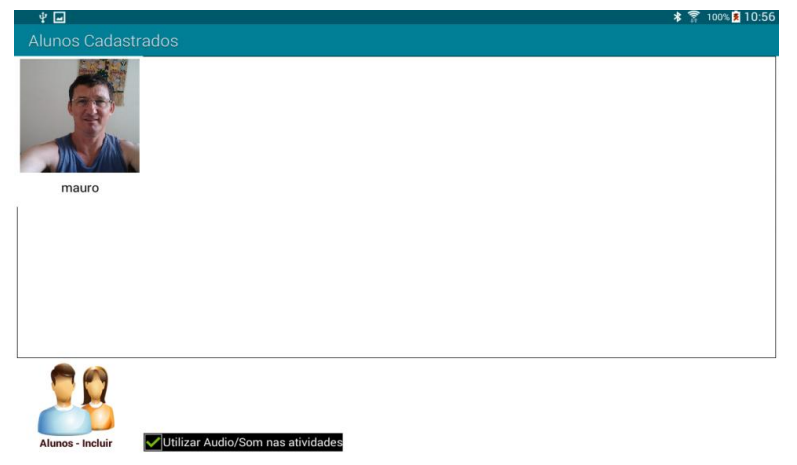

Figura 5-Tela de alunos cadastrados.

A Figura 6 ilustra a interface que é de uso apenas do profissional que realiza o atendimento. Nessa tela o profissional pode entrar sempre que precisar alterar ou registrar os dados do atendimento. Também pode tirar uma fotografia e registrar o áudio relacionado ao aluno. 


\section{CBIE-LACLO 2015}

Anais dos Workshops do IV Congresso Brasileiro de Informática na Educação (CBIE 2015)

$\mathrm{Na}$ atual fase do projeto são disponibilizadas quatro opções de atividades para especialistas e alunos. As atividades do aplicativo são descritas a seguir.

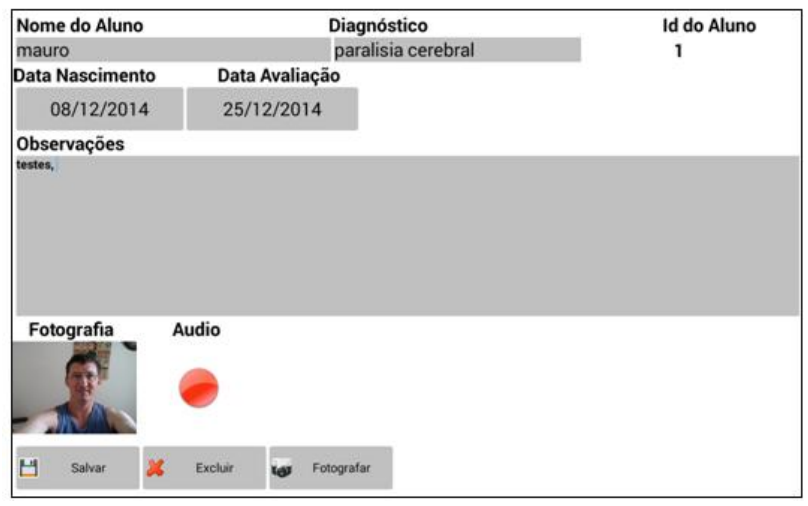

Figura 6-Tela de cadastro do aluno.

A atividade Arraste e Solte tem como objetivo explorar a parte textual e não textual das categorias. A função do aluno é juntar os itens dentro de cada grupo, ver Figura 7. O objetivo dessa atividade é que o aluno selecione os símbolos de cada categoria, que aparecem na parte superior da tela e os coloque dentro de cada caixa, agrupando-os conforme sua relação com cada categoria (Vestuário, Alimentos, Animais, etc|).

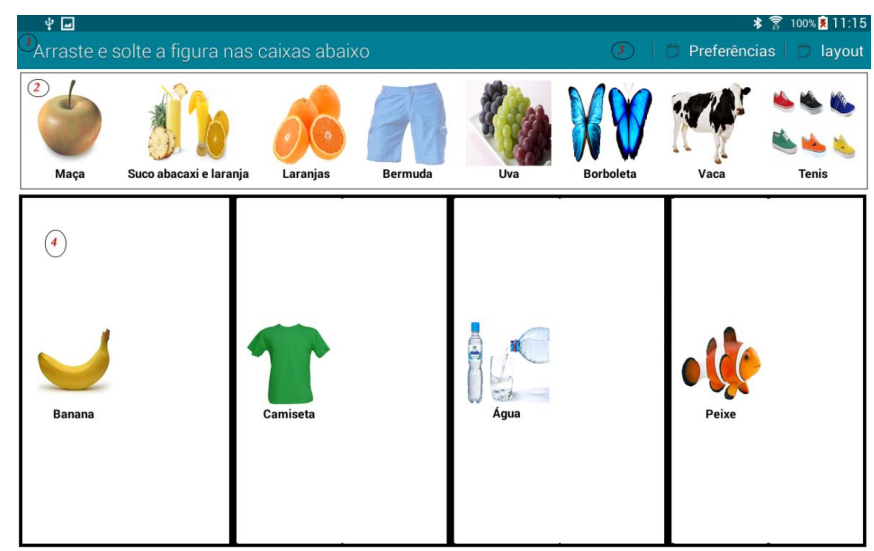

Figura 7-Atividade arrastar e soltar.

Tanto os símbolos ilustrados na parte superior da tela, quanto os símbolos que aparecem na parte inferior da Figura 7, podem ser configurados conforme a preferência do profissional que estiver fazendo o atendimento. Ao final os símbolos devem ser agrupados dentro de suas respectivas categorias, ou não, sendo esta uma das informações necessárias que auxiliam ao profissional a determinar o tipo deficiência intelectual. Salienta-se que todos os símbolos empregados possuem associado um conteúdo textual e também não textual (imagem e áudio) ao ser tocado.

As funcionalidades da atividade Arraste e Solte, são:

1) Barra de título. Descreve resumidamente o conteúdo principal da atividade com texto e áudio.

2) Itens de Categorias selecionadas pelo profissional. Estes itens o aluno deve arrastar e soltar (um item por vez), dentro de sua respectiva categoria. 
3) Preferências e zoom. São opções para o profissional trabalhar a usabilidade na interface ao aluno. Nas preferências ele pode mudar as categorias a serem trabalhadas selecionando as que ele achar necessário para cada etapa do trabalho (de avaliação ou comunicação). Na opção de Zoom, o profissional pode mudar o tamanho dos itens das categorias e a quantidades de caixas (categorias) que vai usar na atividade. Assim pode ser adequado o tamanho e a quantidade de símbolos conforme as características do aluno em estudo.

4) Caixa das categorias. Nesse local os itens ilustrados na parte superior da tela serão arrastados e alocados dentro das respectivas caixas das categorias.

A atividade Acerte o Alvo tem como objetivo analisar a habilidade motora do aluno. O objetivo é que usando um marcador de tempo (temporizador), o software possa avaliar o tempo que aluno leva para selecionar adequadamente o símbolo mostrado, após o primeiro toque na tela, até conseguir o objetivo de acertar o alvo (sobrepor as letras que estão na tela, neste caso, a letra $\mathrm{R}$ laranja coincidindo com a letra $\mathrm{R}$ branca), ver Figura 8. Estas informações poderão ser posteriormente levadas em Conta na Atividade Arraste e solte, de modo a permitir que usuários com problemas motores possam utilizar também o aplicativo, com autonomia.

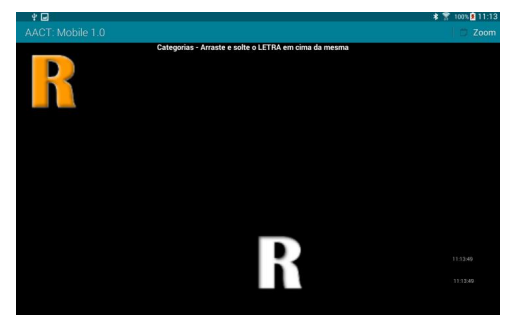

Figura 8 - Acertando o alvo

\section{Características técnicas do aplicativo}

As imagens apresentadas aos alunos no aplicativo foram cedidas pela APAE de Florianópolis no formado em padrões JPEG - Joint Photographic Experts Group e PNG -Portable Network Graphics o que não é a solução ideal para permitir ao gestor realizar livremente alterações nessa configuração. $\mathrm{O}$ mesmo ocorre com outras configurações, tais como o nome e tamanho de fonte dos textos da interface. Entende-se necessário ampliar as possibilidades de configuração em telas próprias do sistema, de modo a fornecer um método que traga, além de homogeneidade, a possibilidade de verificar a consistência dos valores informados.

No aplicativo é utilizado o recurso conhecido como vocalizador. O vocalizador é um recurso eletrônico de gravação/reprodução que ajuda a comunicação das pessoas no cotidiano. Porém, para evitar a voz 'robotizada' o profissional pode gravar sua voz, sendo algo mais agradável e familiar para o aluno. Para o profissional gravar sua voz basta selecionar o botão gravar conteúdo com voz no topo de cada tela.

O dispositivo utilizado no estudo possui uma tela de 10.1 polegadas. O modelo empregado é o Sansung Galaxy Tab4 SM-T530, rodando o sistema operacional Android, API 4.42 (KitKat). A adequação dos conteúdos e imagens no dispositivo segue a orientação de tipo Paisagem (landscape), devido ao melhor enquadramento para os alunos da APAE. Salienta-se que não foi até o presente momento adequado e testado o 


\section{CBIE-LACLO 2015}

Anais dos Workshops do IV Congresso Brasileiro de Informática na Educação (CBIE 2015)

aplicativo para outras interfaces com tamanhos menores a estas especificações, assim como para outras plataformas como Windows Phone e Ios.

\section{Considerações finais}

O trabalho proposto visa contribuir no processo nas atividades de Comunicação Alternativa e Aumentativa de crianças atendidas por profissionais da APAE, através do projeto de uma solução para dispositivos móveis que leva em conta as recomendações de acessibilidade preconizadas pela WMBP. O protótipo também auxilia a avaliar o grau de deficiência intelectual das crianças atendidas, a partir do qual são estabelecidas as ações e metas dos atendimentos. O desenvolvimento também levou em conta os princípios do design centrado no usuário.

Atualmente o trabalho foi avaliado pelos profissionais da APAE desde o mês de Maio, com resultados satisfatórios, podendo ser concluído que o aplicativo possui potencial para atender às necessidades da APAE e das instituições educacionais que recebem alunos com deficiência intelectual e problemas de comunicação. Também está sendo realizada a avaliação do aplicativo, por um especialista em acessibilidade Web. Salienta-se que este trabalho forma parte de uma dissertação de mestrado defendido no mês de Julho.

Em trabalhos futuros, uma das prioridades será o aprimoramento das atividades dos alunos. Considera-se que estas interfaces ainda precisam de alguns ajustes nas suas funcionalidades, sempre observando e levando em conta as recomendações de acessibilidade MWBP e as necessidades das crianças atendidas pela APAE.

\section{Agradecimentos}

Agradecemos a APAE-Florianópolis e a FAPESC, TO. 2015TR300 pelo apoio para o desenvolvimento do projeto.

\section{Referências}

Cook, A. M. and Polgar, J. M. (2008) "Cook And Hussey's Assistive Technologies: Principles And Practice”. St. Louis, Missoouri: Mosby-Elsevier, 2008.

Doval, F. M. G., Carballo, J. M. P. and Jeremías, J. M. V. (2010) Tictac: Information And Communication Technologies For Augmentative Communication Boards. Education Engineering (Educon). Madrid: [S.N.], p. 1783 - 1787.

Higashi, T. et al. (2005) Nonverbal Communication Tool For Children With Severe Motor And Intellectual Disabilities Using Biochemical Measurement. 27th Annual International Conference Of The Engineering In Medicine And Biology Society. Shanghai: [S.N.], p. 3538 - 3541. Proceedings.

Rabin, J. and Mccathienevile, C. (2008) "Mobile Web Best Practices 1.0.W3C". <http://Www.W3.Org/Tr/Mobile-Bp/>. Maio de 2014.

Schindler, K., Dygert, R. and Nagler, E. (2009) A reconfigurable augmentative communication device. 11th international ACM SIGACCESS conference on Computers and accessibility. New York: [s.n.], p. 217-218 